\title{
On Solution of Integrodifferential Equation with Delay Parameter by Sinc Basis Functions
}

\author{
I. Najafi Khalilsaraye and K. Maleknejad \\ Department of Mathematics, College of Basic Sciences, Karaj Branch, Islamic Azad University, Alborz, Iran \\ Correspondence should be addressed to K. Maleknejad; maleknejad@iust.ac.ir
}

Received 10 March 2014; Accepted 18 April 2014; Published 8 May 2014

Academic Editor: Reza Ezzati

Copyright ( 2014 I. Najafi Khalilsaraye and K. Maleknejad. This is an open access article distributed under the Creative Commons Attribution License, which permits unrestricted use, distribution, and reproduction in any medium, provided the original work is properly cited.

\begin{abstract}
We want to find a numerical solution for an integrodifferential equation with an integral boundary condition and delay parameter. This type of problems arises in mathematical physics, mechanics, population growth, and other fields of physics and mathematical chemistry. So, convergence of this approach is discussed by presenting a theorem which gives exponential type convergence rate and guarantees the accuracy of that. Finally, by some numerical examples, we show the efficiency and accuracy of this numerical method.
\end{abstract}

\section{Introduction}

Discussing integrodifferential equations with integral boundary condition consisting of delay parameter is a worthy and significant branch of nonlinear applied mathematics. It is important that integrodifferential equation with delay parameter is generated often in investigations connected with chemical engineering, mathematical physics, underground water flow, engineering, and so on (see $[1,2])$. Note that the problems with integral boundary conditions have various applications in applied fields such as population growth problems and blood flow problems. For a detailed description of the integrodifferential equations with delay parameter and problems with integral boundary conditions, the reader can refer to references of [3-7].

In this paper we discuss the following problem which shows a first-order integrodifferential equation with integral boundary condition consisting of delay parameter. The existence and uniqueness of solution for this problem are proved in [8]. But analytic solving and reaching an exact solution are impossible. Then in this paper we approximate the exact solution by numerical method

$$
\begin{gathered}
\frac{d y}{d x}=g\left(x, y(x), y\left(\gamma_{1}(x)\right), U y(x), V y(x)\right) \equiv G y(x), \\
y(0)=\eta_{1} y(\kappa)+\eta_{2} \int_{0}^{b} \omega(r, y(r)) d r+a,
\end{gathered}
$$

where $x \in I=[0, b](b>0), g \in C\left(I \times \mathbb{R}^{4}, \mathbb{R}\right)$, where $C$ is family of all continuous functions, and $\gamma_{1} \in C(I, I), \kappa \in(0, b]$, $\omega \in C(I \times \mathbb{R}, \mathbb{R}), \eta_{1}, \eta_{2}, a \in \mathbb{R}$.

And

$$
\begin{gathered}
(U y)(x)=\int_{0}^{\gamma_{2}(x)} k(x, r) y(B(r)) d r, \\
(V y)(x)=\int_{0}^{b} h(x, r) y(D(r)) d r .
\end{gathered}
$$

Here $\gamma_{2}, B, D \in C(I, I), k(x, r) \in C\left[A, \mathbb{R}^{+}\right]$, and $h(x, r) \in$ $C\left[A_{0}, \mathbb{R}^{+}\right]$that

$$
\begin{gathered}
A=\left\{(x, r) \in \mathbb{R}^{2} \mid 0 \leq r \leq \gamma_{2}(x), x \in I\right\}, \\
A_{0}=\left\{(x, r) \in \mathbb{R}^{2} \mid 0 \leq r \leq x, x \in I\right\} .
\end{gathered}
$$

Here if $\eta_{1}=1, \eta_{2}=a=0$, and $\kappa=b$, then we have a problem with boundary condition of kind periodic, and if $\eta_{1}=0$, then we have a problem with integral boundary condition, and if $\eta_{1}=\eta_{2}=0$, we have a problem with an initial condition. So, problem (1) is general type of these cases. 
Now, the following functional integral equation can be easily concluded from (1):

$$
\begin{gathered}
y(x)=\eta_{1} y(\kappa)+\eta_{2} \int_{0}^{b} \omega(r, y(r)) d r+a \\
+\int_{0}^{x} g\left(t, y(t), y\left(\gamma_{1}(t)\right), \int_{0}^{\gamma_{2}(t)} k(t, r) y(B(r)) d r,\right. \\
\left.\int_{0}^{b} h(t, r) y(D(r)) d r\right) d t
\end{gathered}
$$

and in this paper we want to discuss solution of (1) in point of equivalent integral equation (4). But we know the fact that we cannot solve this integral equation to give an exact solution, so numerical approaches are used to reach an approximated solution.

Numerical approaches for estimated solution of integrodifferential and integral equation and search for existence and uniqueness of solution for some problems have been researched by many authors and reader can see these methods in [9-15]. In these references authors use methods on an estimate by basis function such as wavelets, polynomials, and so forth or use some quadrature formulas. But these technics usually have convergence rate of polynomial order with respect to $M$ where $M$ represents the cardinal of terms of sum in the expansion or the cardinal of points of the quadrature formula. In [16] author showed that if we employ the Sinc approach, the convergence rate is exponential order such as $O\left(\exp \left(-c M^{1 / 2}\right)\right)$ with some $c>0$. We know the exponential rate is much faster than that of polynomial rate. So, in this paper, we employ the Sinc function instead of base function and an iterative technique to estimate exact solution of (4) in marked points. Our approach dose not contain of changing the solution of (4) to a system of algebraic equations by expanding $y(x)$ as basis function with unknown coefficients, so this technique has computations less than other methods and exponential rate in accuracy. Also in this present paper, we prove a theorem to guarantee the convergence of numerical technique.

\section{Main Results}

In this section, we introduce basic requirements and theorem to prove existence and uniqueness of solution for firstorder integrodifferential equation with integral boundary condition consisting of delay parameter (1). For detailed descriptions, we refer the reader to $[17,18]$.

Definition 1. A function as $y \in C^{1}(I, I)$ is called a lower solution of (1) if

$$
\begin{gathered}
\frac{d y}{d x} \leq G y(x), \quad x \in I, \\
y(0) \leq \eta_{1} y(\kappa)+\eta_{2} \int_{0}^{b} \omega(r, y(r)) d r+a
\end{gathered}
$$

and it is an upper solution of (1) if

$$
\begin{gathered}
\frac{d y}{d x} \geq G y(x), \quad x \in I, \\
y(0) \geq \eta_{1} y(\kappa)+\eta_{2} \int_{0}^{b} \omega(r, y(r)) d r+a .
\end{gathered}
$$

Theorem 2. Let $u_{0}, v_{0} \in C^{1}(I, \mathbb{R})$ be lower and upper solutions of (1), respectively, and $u_{0}(x) \leq v_{0}(x), x \in I$.

In addition consider the following.

$\left(L_{1}\right): g \in C\left(I \times \mathbb{R}^{4}, \mathbb{R}\right), \gamma_{1}, \gamma_{2}, B, D \in C(I, I), \gamma_{1}(x), \gamma_{2}(x)$, $B(x), D(x) \leq x, \forall x \in I, \kappa \in(0, b), \omega \in C(I \times \mathbb{R}, \mathbb{R})$, and $\eta_{1}, \eta_{2} \geq 0$.

$\left(L_{2}\right)$ : there are nonnegative bounded integrable functions $M_{1}(x), M_{2}(x), M_{3}(x), M_{4}(x)$ on I that

$$
\begin{gathered}
\int_{0}^{b}\left[M_{1}(t)+M_{2}(x)+M_{3}(x) \int_{0}^{\gamma_{2}(x)} k(x, r) d r\right. \\
\left.+M_{4}(x) \int_{0}^{b} h(x, r) d r\right] d x \leq 1
\end{gathered}
$$

such that

$$
\begin{gathered}
g\left(x, \phi_{1}, \phi_{2}, U \phi_{1}, V \phi_{1}\right)-g\left(x, \psi_{1}, \psi_{2}, U \psi_{1}, V \psi_{1}\right) \\
\geq-M_{1}(x)\left(\phi_{1}-\psi_{1}\right)-M_{2}(x)\left(\phi_{2}-\psi_{2}\right) \\
\quad-M_{3}(x) U\left(\phi_{1}-\psi_{1}\right)-M_{4}(x) V\left(\phi_{1}-\psi_{1}\right)
\end{gathered}
$$

if $u_{0} \leq \psi_{1} \leq \phi_{1} \leq v_{0}, u_{0}\left(\gamma_{1}(x)\right) \leq \psi_{2} \leq \phi_{2} \leq v_{0}\left(\gamma_{1}(x)\right)$.

$\left(L_{3}\right)$ : there is $\theta(x) \in C\left(I, \mathbb{R}^{+}\right)$such that $\omega(x, \psi)-\omega\left(x, \psi^{-}\right) \geq$ $\theta(x)\left(\psi-\psi^{-}\right)$, and if $u_{0}(t) \leq \psi^{-} \leq \psi \leq v_{0}(x)$. then problem (1) has extremal solutions $u, v \in\left[u_{0}, v_{0}\right]$. In addition, there are monotone sequences $u_{n}(x), v_{n}(x) \subset$ $\left[u_{0}, v_{0}\right]$ such that $u_{n} \rightarrow u, v_{n} \rightarrow v$ for $n \rightarrow+\infty$ and these are convergent uniformly on $x \in I$, where $u_{n}(x)$, $v_{n}(x)$ are defined as

$$
\begin{aligned}
& u_{n}(x)=\int_{0}^{x} e^{-\int_{r}^{x} M_{1}(s) d s} \\
& \times\left[g \left(r, u_{n-1}(r), u_{n-1}\left(\gamma_{1}(r)\right),\right.\right. \\
& \left.U u_{n-1}(r), V u_{n-1}(r)\right)+M_{1}(r) u_{n-1}(r) \\
& -M_{2}(r)\left(u_{n}-u_{n-1}\right)\left(\gamma_{1}(r)\right) \\
& -M_{3}(r) U\left(u_{n}-u_{n-1}\right)(r) \\
& \left.-M_{4}(r) V\left(u_{n}-u_{n-1}\right)(r)\right] d r \\
& +e^{-\int_{0}^{x} M_{1}(s) d s}\left[\eta_{1} u_{n-1}(\kappa)\right. \\
& \left.+\eta_{2} \int_{0}^{b} \omega\left(r, u_{n-1}(r)\right) d r+a\right] \\
& \forall x \in I, \quad n=1,2,3, \ldots,
\end{aligned}
$$




$$
\begin{aligned}
& v_{n}(x)=\int_{0}^{x} e^{-\int_{r}^{x} M_{1}(s) d s} \\
& \times\left[g \left(r, v_{n-1}(r), v_{n-1}\left(\gamma_{1}(r)\right),\right.\right. \\
& \left.U v_{n-1}(r), V v_{n-1}(r)\right)+M_{1}(r) z_{n-1}(r) \\
& -M_{2}(r)\left(v_{n}-v_{n-1}\right)\left(\gamma_{2}(r)\right) \\
& -M_{3}(r) U\left(v_{n}-v_{n-1}\right)(r) \\
& \left.-M_{4}(r) V\left(v_{n}-v_{n-1}\right)(r)\right] d r \\
& +e^{-\int_{0}^{x} M_{1}(s) d s}\left[\eta_{1} v_{n-1}(\kappa)\right. \\
& \left.+\eta_{2} \int_{0}^{b} \omega\left(r, v_{n-1}(r)\right) d r+a\right] \\
& \forall x \in I, \quad n=1,2,3, \ldots, \\
& u_{0} \leq u_{1} \leq \cdots \leq u_{n} \leq \cdots \leq u \leq v \leq \cdots \leq v_{n} \\
& \leq \cdots \leq v_{1} \leq v_{0} .
\end{aligned}
$$

Proof. See [18].

Theorem 3. Consider that assumptions of Theorem 2 hold. Moreover, consider the following.

$\left(L_{4}\right)$ : there are nonnegative bounded functions $\alpha_{1}(x), \alpha_{2}(x)$, $\alpha_{3}(x), \alpha_{4}(x)$ on $I$, such that

$$
\begin{aligned}
& g\left(x, \phi_{1}, \phi_{2}, U \phi_{1}, V \phi_{1}\right)-g\left(x, \psi_{1}, \psi_{2}, U \psi_{1}, V \psi_{1}\right) \\
& \leq \alpha_{1}(x)\left(\phi_{1}-\psi_{1}\right)+\alpha_{2}(x)\left(\phi_{2}-\psi_{2}\right) \\
&+\alpha_{3}(x) U\left(\phi_{1}-\psi_{1}\right)+\alpha_{4}(x) V\left(\phi_{1}-\psi_{1}\right)
\end{aligned}
$$

if $u_{0}(x) \leq \psi_{1} \leq \phi_{1} \leq v_{0}(x), u_{0}\left(\gamma_{1}(x)\right) \leq \psi_{2} \leq \phi_{2} \leq$ $v_{0}\left(\gamma_{1}(x)\right)$.

$\left(L_{5}\right)$ : there is $\beta(x) \in \mathscr{C}\left(I, \mathbb{R}^{+}\right)$such that $\omega(x, \psi)-\omega\left(x, \psi^{-}\right) \leq$ $\beta(x)\left(\psi-\psi^{-}\right)$if $u_{0}(t) \leq \psi^{-} \leq \psi \leq v_{0}(x)$.

Then problem (1) has a unique solution $u^{-} \in\left[u_{0}, v_{0}\right]$. In addition, there are sequences $u_{n}(x), v_{n}(x) \subset\left[u_{0}, v_{0}\right]$ that these are monotone and $u_{n} \rightarrow u^{-}, v_{n} \rightarrow u^{-}$ for $n \rightarrow+\infty$. This convergence is uniformly on $x \in$ $I$, where $u_{n}(x), v_{n}(x)$ are defined as (9) such that $\left\|u_{n}-u^{-}\right\|_{c} \leq L^{n}\left\|v_{0}-u_{0}\right\|_{c}, n \in \mathbb{N}$, where

$$
\begin{aligned}
L=\eta_{1}+\int_{0}^{b}\left[\eta_{2} \beta(x)+\alpha_{1}(x)+M_{1}(x)+\alpha_{2}(x)+M_{2}(x)\right. \\
+\left(\alpha_{3}(x)+M_{3}(x)\right) \int_{0}^{\gamma_{2}(x)} K(x, r) d r \\
\left.+\left(\alpha_{4}(x)+M_{4}(x)\right) \int_{0}^{b} h(x, r) d r\right] d x<1 .
\end{aligned}
$$

Proof. See [18].

\section{Sinc Function}

In this section, we recall the basis function and some of its applicabilities. In here, definition of $\operatorname{sinc}(x)$ function is followed by

$$
\operatorname{Sinc}(x)= \begin{cases}\frac{\sin (\pi x)}{\pi x} ; & x \neq 0, \\ 1 ; & x=0 .\end{cases}
$$

Now, for $h>0$ and integer $j$, we define $j$ th Sinc function with step size $h$ by

$$
S(j, h)(x)=\frac{\sin (\pi(x-j h) / h)}{\pi(x-j h) / h} .
$$

3.1. Sinc Estimation on $[a, b]$. Let $x=\varphi(w)$ be a transformation that denotes a conformal transformation which transfers the simply connected domain $A$ onto a strip region $A_{d}$ such that

$$
\begin{gathered}
\varphi((a, b))=(-\infty, \infty), \quad \lim _{x \rightarrow a} \varphi(x)=-\infty, \\
\lim _{x \rightarrow b} \varphi(x)=\infty .
\end{gathered}
$$

In here $\partial A$ is boundary of $A$ and in order to have the Sinc estimation on $(a, b)$ conformal transformation is applied as follows:

$$
\varphi(t)=\ln \left(\frac{t-a}{b-t}\right)
$$

This function transfers the eye-shaped complex region

$$
\left\{w=x+i y:\left|\arg \left(\frac{w-a}{b-w}\right)\right|<d \leq \frac{\pi}{2}\right\}
$$

onto $A_{d}$ that it is a strip region:

$$
A_{d}=\left\{\sigma=\alpha+\beta i:|\beta|<d<\frac{\pi}{2}\right\} \text {. }
$$

The basis functions on finite interval $(a, b)$ are given by

$$
S(j, h) \circ \varphi(x)=\frac{\sin (\pi(\varphi(x)-j h) / h)}{\pi(\varphi(x)-j h) / h},
$$

and also, Sinc function for interpolation points $x_{j}=j h$ is given by

$$
S(j, h)(k h)=\delta_{j k}^{(0)}= \begin{cases}1 ; & j=k, \\ 0 ; & j \neq k .\end{cases}
$$

Then, $S(j, h) \circ \varphi(x)$ shows behavior of Kronecker delta function on the network points

$$
x_{j}=\varphi^{-1}(j h)=\frac{a+b e^{j h}}{1+e^{j h}} .
$$


The approximation of $g(x)$ by interpolation and quadrature formulas for $\int_{a}^{b} g(x) d x$ is

$$
\begin{gathered}
g(x) \approx \sum_{j=-M}^{M} g\left(x_{j}\right) S(j, h) \circ \varphi(x), \\
\int_{a}^{b} g(x) d x \approx h \sum_{j=-M}^{M} \frac{g\left(x_{j}\right)}{\varphi^{\prime}\left(x_{j}\right)} .
\end{gathered}
$$

Theorem 4. Consider that, for a map $w=\varphi^{-1}(\xi)$, the map $g\left(\varphi^{-1}(\xi)\right)$ satisfies

(1) $g \in H^{1}\left(D_{d}\right)$, for $d>0$,

(2) $g$ decays exponentially on the real line such that

$$
|g(x)| \leq \alpha \exp (-\beta|x|), \quad \forall x \in \mathbb{R}, \alpha, \beta>0
$$

with some $\alpha, \beta$, and $d$. Then one has

$$
\begin{aligned}
& \sup _{a<x<b}\left|g(x)-\sum_{j=-M}^{M} g\left(\varphi^{-1}(j h)\right) S(j, h) \circ \varphi(x)\right| \\
& \leq C \sqrt{M} \exp (-\sqrt{\pi d \beta M}) .
\end{aligned}
$$

That there is some $C$ and $h$ is $h=\sqrt{\pi d / \beta M}$.

Proof. See [16].

Definition 5. Let $L_{\alpha}(A)$ be the set of all analytic functions $g$, for which there exists a constant $C$, such that

$$
|g(z)| \leq C \frac{\left|e^{\varphi(z)}\right|^{\alpha}}{\left(1+\left|e^{\varphi(z)}\right|\right)^{2 \alpha}} ; \quad z \in A, 0<\alpha \leq 1
$$

Theorem 6. Let $g / \varphi^{\prime} \in L_{\alpha}(A)$, with $0<\alpha \leq 1$ and $0<d \leq \pi$; also let $h=\sqrt{\pi d / \alpha M}$. Then there exists a constant $C_{1}$, which is independent of $M$, such that

$$
\left|\int_{a}^{x_{j}} g(t) d t-h \sum_{k=-M}^{M} \delta_{j k}^{(-1)} \frac{g\left(x_{j}\right)}{\varphi^{\prime}\left(x_{k}\right)}\right| \leq C_{1} e^{-\sqrt{\pi d \alpha M}},
$$

where

$$
\delta_{j k}^{(-1)}=\frac{1}{2}+\int_{0}^{j-k} \frac{\sin (\pi t)}{\pi t} d t
$$

and $\varphi(x), x_{k}$ are defined as above.

Proof. See [16].
3.2. Sinc-Qudrature Method. In this section, for solving equation

$$
\begin{array}{r}
y(x)=\lambda_{1} y(\kappa)+\eta_{2} \int_{0}^{b} \omega(r, y(r)) d r+a \\
+\int_{0}^{x} g\left(s, y(s), y\left(\gamma_{1}(s)\right),\right. \\
\quad \int_{0}^{\gamma_{2}(s)} k(s, r) y(B(r)) d r, \\
\left.\int_{0}^{b} h(s, r) y(D(s)) d r\right) d s,
\end{array}
$$

we try to discrete integral equation by quadrature formula as

$$
\int_{a}^{b} g(s) d s=h \sum_{j=-M}^{M} \frac{g\left(s_{j}\right)}{\varphi^{\prime}\left(s_{j}\right)}+O\left(\exp \left(-\frac{2 \pi d M}{\log (2 \pi d M / \beta)}\right)\right)
$$

$$
\begin{aligned}
\int_{a}^{s} f(x) d x= & h \sum_{j=-M}^{M} \frac{g\left(x_{j}\right)}{\varphi^{\prime}\left(x_{j}\right)} \eta_{h, j}(s) \\
& +O\left(\frac{\log M}{M} \exp \left(-\frac{\pi d M}{\log (\pi d M / \beta)}\right)\right),
\end{aligned}
$$

where

$$
\eta_{h, j}(s)=\frac{1}{2}+\frac{1}{\pi} r_{i}\left(\pi \frac{r-j h}{h}\right) ; \quad r_{i}=\int_{0}^{x} \frac{\sin (t)}{t} d t
$$

with $x_{j}=s_{j}=\left(a+b e^{j h}\right) /(1+j h), j=-M, \ldots, M$ and $h=$ $(1 / M) \log (\pi d M / \beta)($ see $[16])$

Now, by substituting quadrature formulas in the integral equation (4), we have

$$
\begin{gathered}
y_{M}(x)=\eta_{1} y(\kappa)+\eta_{2} h \sum_{j=-M}^{M} \frac{\omega\left(s_{j}, y\left(s_{j}\right)\right)}{\varphi^{\prime}\left(s_{j}\right)}+a \\
+h \sum_{j=-M}^{M} g\left(s_{j}, y\left(s_{j}\right), y\left(\alpha\left(s_{j}\right)\right),\right. \\
h \sum_{i=-M}^{M} \frac{K\left(s_{j}, r_{i}\right) y\left(B\left(r_{i}\right)\right)}{\varphi^{\prime}\left(r_{i}\right)} \eta_{h, i}(t), \\
\left.h \sum_{i=-M}^{M} \frac{h\left(s_{j}, r_{i}\right) y\left(D\left(r_{i}\right)\right)}{\varphi^{\prime}\left(r_{i}\right)}\right) \\
\times\left(\varphi^{\prime}\left(s_{j}\right)\right)^{-1} \eta_{h, j}(x) .
\end{gathered}
$$


Now, for $n=1,2,3, \ldots$, let

$$
y_{1, M}(x)=\eta_{1} y(\kappa)
$$

$$
y_{n+1, M}(x)
$$

$$
\begin{gathered}
=\eta_{1} y_{n, M}(\kappa)+\eta_{2} h \sum_{j=-M}^{M} \frac{\omega\left(s_{j}, y_{n, M}\left(s_{j}\right)\right)}{\varphi^{\prime}\left(s_{j}\right)}+a \\
+h \sum_{j=-M}^{M} g\left(s_{j}, y_{n, M}\left(s_{j}\right), y_{n, M}\left(\alpha\left(s_{j}\right)\right),\right. \\
h \sum_{i=-M}^{M} \frac{a\left(s_{j}, r_{i}\right) y_{n, M}\left(B\left(r_{i}\right)\right)}{\varphi^{\prime}\left(r_{i}\right)} \eta_{h, i}(x), \\
\left.\quad h \sum_{i=-M}^{M} \frac{h\left(s_{j}, r_{i}\right) y_{n, M}\left(\delta\left(r_{i}\right)\right)}{\varphi^{\prime}\left(r_{i}\right)}\right) \\
\times\left(\varphi^{\prime}\left(s_{j}\right)\right)^{-1} \eta_{h, j}(x) .
\end{gathered}
$$

\section{Convergence of Method}

In this section, we present a theorem that shows a bound for $y(x)-y_{n}(x)$ with the real norm where $y(x)$ is the exact solution of problem (4) and $y_{n}(x)$ is an estimation for $y(x)$ by using Sinc function in interpolaion and quadrature formula. The result is shown as follows.

Theorem 7. Under the assumptions $\left(L_{1}\right)-\left(L_{5}\right)$, iterative approximation approach (31) is convergent to exact solution if $y_{1}(x)$ is closed enough to the it and

$$
N_{2} \leq \frac{1-\left(\eta_{1}+\eta_{2} b N_{1}\right)}{b\left(2+N_{3}+N_{4}\right)},
$$

where $N_{1}=\sup \{\beta(x) ; x \in I\}, N_{2}=\max \left\{\alpha_{i}(x) ; i=1,2,3,4\right.$, $x \in I\}, N_{3}=\sup \{K(x, r) ; x, r \in I\}$, and $N_{4}=\sup \{h(x, r)$; $x, r \in I\}$.

Proof. For a fixed $M$ let

$$
\begin{aligned}
& y_{n+1, M}(x)= \eta_{1} x_{n, M}(\tau)+\eta_{2} \int_{0}^{b} \omega\left(r, y_{n, M}(r)\right) d r+a \\
&+\int_{0}^{x} g\left(s, y_{n, M}(s), y_{n, M}\left(\gamma_{1}(s)\right), U y_{n, M}(s),\right. \\
&\left.V y_{n, M}(s)\right) d s \\
& y(x)=\eta_{1} y(\kappa)+\eta_{2} \int_{0}^{b} \omega(r, y(r)) d r+a \\
&+\int_{0}^{x} g\left(s, x(s), y\left(\gamma_{1}(s)\right), U y(s), V y(s)\right) d s .
\end{aligned}
$$

Now

$$
\begin{aligned}
& \left|y_{n+1, M}(x)-y(x)\right| \\
& \leq \eta_{1}\left|y_{n}(\kappa)-y(\kappa)\right| \\
& +\eta_{2}\left|\int_{0}^{b}\left(\omega\left(r, y_{n}(r)\right)-\omega(r, y(r))\right) d r\right| \\
& +\mid \int_{0}^{x}\left[g \left(s, y_{n}(s), y_{n}\left(\gamma_{1}(s)\right),\right.\right. \\
& \int_{0}^{\gamma_{2}(s)} K(s, r) y_{n}(B(r)) d r, \\
& \left.\int_{0}^{b} h(s, r) y_{n}(D(r)) d r\right) \\
& -f\left(s, y(s), y\left(\gamma_{1}(s)\right),\right. \\
& \int_{0}^{\gamma_{2}(s)} K(s, r) y(B(r)) d r, \\
& \left.\left.\int_{0}^{b} h(s, r) y(D(r)) d r\right)\right] d s \mid \\
& \leq \eta_{1}\left|y_{n}(\kappa)-y(\kappa)\right|+\eta_{2} b N_{1}\left|y_{n}(x)-y(x)\right| \\
& +b N_{2}\left|y_{n}(x)-y(x)\right|+b N_{2}\left|y_{n}\left(\gamma_{1}(x)\right)-y\left(\gamma_{1}(x)\right)\right| \\
& +b N_{2} N_{3}\left|x_{n}(B(r))-y(B(r))\right| \\
& +b N_{2} M_{4}\left|y_{n}(D(r))-y(D(r))\right| \\
& \leq\left(\eta_{1}+\eta_{2} b N_{1}+b N_{2}\left(2+N_{3}+N_{4}\right)\right)\left|y_{n, M}(x)-y(x)\right| .
\end{aligned}
$$

Then

$$
\begin{aligned}
\left|y_{n+1, M}(x)-y(x)\right| \leq & \left(\eta_{1}+\eta_{2} b N_{1}+b N_{2}\left(2+N_{3}+N_{4}\right)\right)^{n} \\
& \times\left|y_{1, M}(x)-y(x)\right| .
\end{aligned}
$$

Now, by assumption in theorem, we have $\eta_{1}+\eta_{2} b N_{1}+b N_{2}(2+$ $\left.N_{3}+N_{4}\right)<1$.

Because $\eta_{1}+\eta_{2} b N_{1}+b N_{2}\left(2+N_{3}+N_{4}\right)<\eta_{1}+\eta_{2} b N_{1}+$ $b\left(\left(1-\left(\eta_{1}+\eta_{2} b N_{1}\right)\right) / b\left(2+N_{3}+N_{4}\right)\right)\left(2+N_{3}+N_{4}\right)=1$ and then $\lim _{n \rightarrow+\infty} y_{n+1, M}(x)=y(x), 0 \leq x \leq b$.

\section{Illustrative Examples}

In this section, for showing efficiency of the iterative scheme and in order to show the facts of the exact solution, we give some examples below. All routines have been written in Mathematica 7 and a Dual-Core CPU $2.00 \mathrm{GHz}$ is used to run the programs. Also, about efficiency and accuracy of the proposed numerical method, we present absolute errors for different examples. Therefore numerical results are shown in figures to illustrate the efficiency of this scheme. 


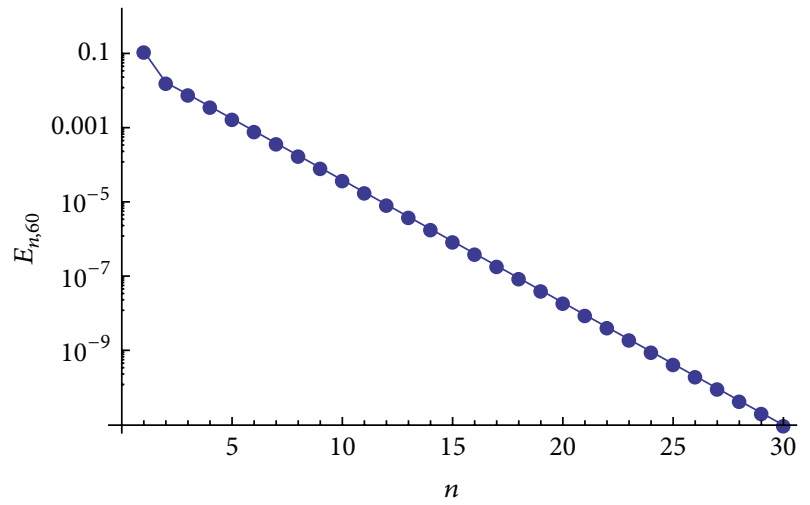

FIgURE 1: Absolute errors of Example 1. For different values of iteration $n$.

Example 1. Consider the following problem:

$$
\begin{aligned}
\frac{d y}{d x}= & \frac{1}{15} x^{3}[x-y(x)]-\frac{1}{5} x y\left(\gamma_{1}(x)\right)+\frac{1}{100} x^{2} y^{2}\left(\gamma_{1}(x)\right) \\
& +\frac{1}{500} x\left[x^{3}-\int_{0}^{(1 / 2) x} 8 x r y\left(r^{2}\right) d r\right]^{5} \\
& -\frac{1}{600} x^{2}\left[x^{3}-\int_{0}^{(1 / 2) x} 8 x r y\left(r^{2}\right) d r\right]^{6} \\
& +\frac{1}{700} x^{2}\left[x^{2}-\int_{0}^{1} 2 x^{2} r y\left(r^{2}\right) d r\right]^{7} \\
& -\frac{1}{800} x^{3}\left[x^{2}-\int_{0}^{1} 2 x^{2} r y\left(r^{2}\right) d r\right]^{8} \equiv G y(x), \\
& y(0)=\frac{1}{4} y(\kappa)+\frac{1}{4} \int_{0}^{1}(2 r+y(r)) d r+\frac{1}{4},
\end{aligned}
$$

where $\kappa \in(0,1], \gamma_{1} \in C(I, I)$, and $\gamma_{1}(x) \leq x$ on $I$.

Now, we want to show that assumptions $\left(L_{1}\right)-\left(L_{5}\right)$ and convergence criterion which was prepared in Theorem 7 are satisfied for integral equation (4).

Noting that $\gamma_{2}(x)=(1 / 2) x, B(x)=D(x)=x^{2}, \forall x \in I$, then $\left(L_{1}\right)-\left(L_{5}\right)$ is true. For details see [6].

In addition, we have $\beta(x)=1, \alpha_{1}(x)=0, \alpha_{2}(x)=$ $(1 / 50) x^{2}, \alpha_{3}(x)=\alpha_{4}(x)=(1 / 100) x^{17}, K(x, r)=8 x r$, $h(x, r)=2 x^{2} r$, and $\eta_{1}=\eta_{2}=1 / 4$; then $N_{1}=1, N_{2}=1 / 50$, $N_{3}=8$, and $N_{4}=2$, and then

$$
N_{2}=\frac{1}{50} \leq \frac{1-\left(\eta_{1}+\eta_{2} b N_{1}\right)}{b\left(2+N_{3}+N_{4}\right)}=\frac{1}{24} \text {. }
$$

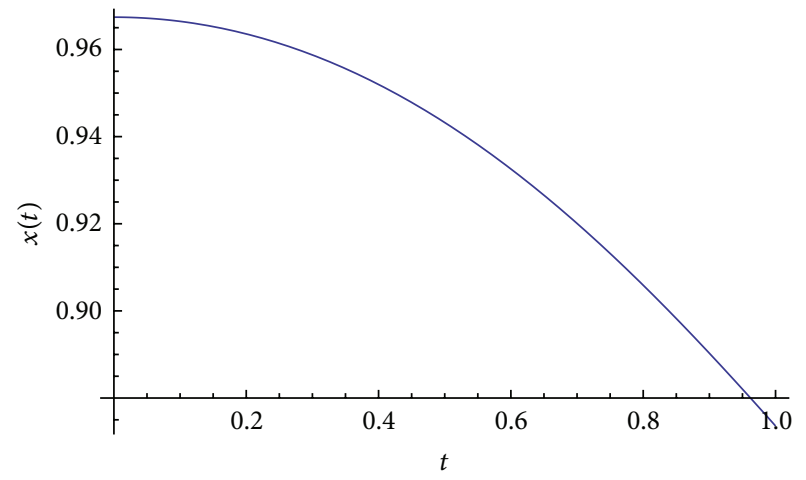

FIgURe 2: Approximate solution for Example 1.

Therefore, the iterative method (31) converges to the exact solution of this equation. Now, based on Sinc quadrature scheme we can have some successive approximations for solution of this example for $\kappa=1 / 2$. Absolute error for $n$th approximation and $N$ points quadrature method is defined by

$$
e_{n, M}=\max _{x \in[0,1]}\left\{\left|y_{n+1, M}(x)-y_{n, M}(x)\right|\right\} ; \quad n=1,2,3, \ldots
$$

For different values of $n$ absolute errors are depicted in Figure 1 and approximate solution for $n=30$ is depicted in Figure 2.

Example 2. In Example 1 of [19], authors considered the integrodifferential equation

$$
\begin{gathered}
\frac{d y}{d x}=1-\frac{1}{3} x^{3}+\int_{0}^{1} x^{3} y^{2}(z) d z \\
y(0)=0 .
\end{gathered}
$$

The exact solution is $y(x)=x$. Maximum absolute error for each iteration and different values of quadrature points are depicted in Figures 3 and 4. By comparing these results with the numerical results given in [19] in Table 2, efficiency and accuracy of current approach are guaranteed.

\section{Conclusion}

In this paper, we apply a numerical approach by Sinc function for reaching the estimated solution of integrodifferential equation with enteral boundary condition and with delay parameter. To reach this aim we change this problem to a functional enteral equation. The Sinc estimation has exponential convergence rate such as $O\left(-c e^{M^{1 / 2}}\right)$ that this property is an advantage. so we applied it to solve our problem by using collocation method. Finally, some examples are solved by this numerical method to show the efficiency and accuracy of Sinc estimation. It is worthy to note that this method can 


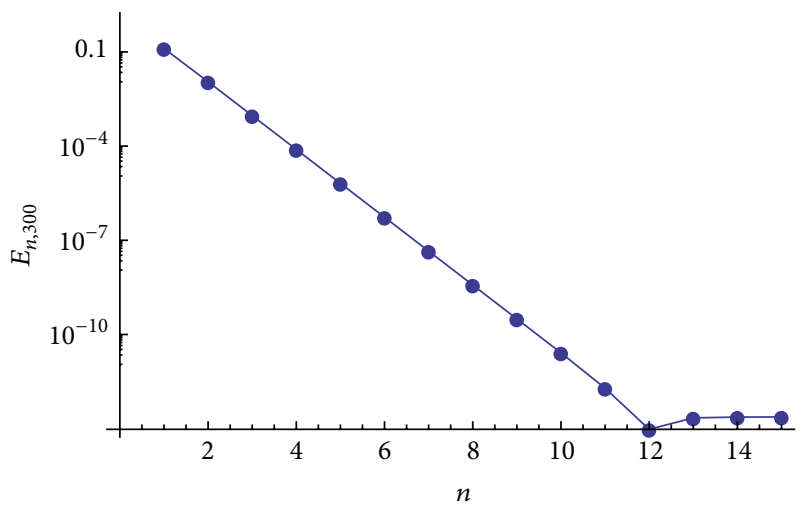

FIGURE 3: Maximum absolute error related to each iteration in Example 2.

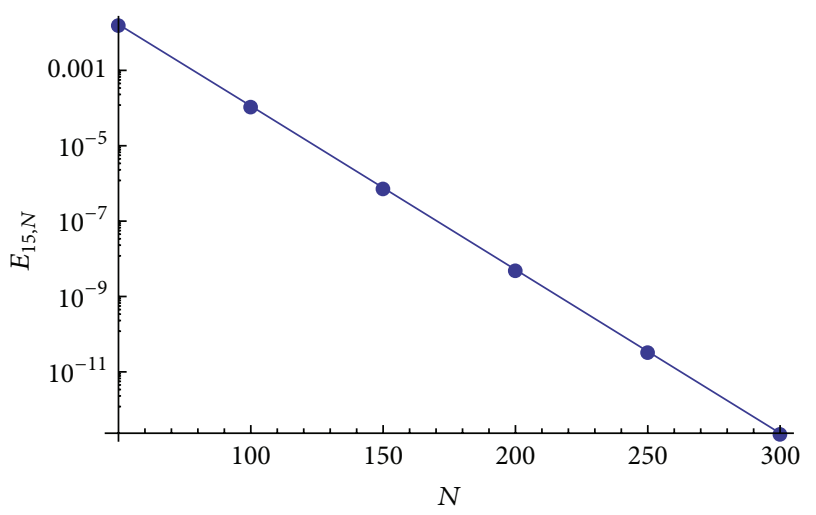

FIGURE 4: Maximum error related to different quadrature points in Example 2.

be used for solving integrodifferential equations with integral boundary conditions with deviating arguments arising in all sciences such as chemistry, physics, and other fields of applied mathematics.

\section{Conflict of Interests}

The authors declare that there is no conflict of interests regarding the publication of this paper.

\section{Acknowledgment}

The authors thank the referee for his/her careful reading of the paper and useful suggestion.

\section{References}

[1] R. P. Agarwal, D. O’Regan, and P. J. Y. Wong, Positive Solutions of Differential, Difference and Integral Equations, Kluwer Academic Publishers, Dordrecht, The Netherlands, 1999.
[2] T. A. Burton, "Differential inequalities for integral and delay differential equations," in Comparison Methods and Stability Theory, vol. 162 of Lecture Notes in Pure and Applied Mathematics, pp. 35-56, Dekker, New York, NY, USA, 1994.

[3] B. Ahmad and B. S. Alghamdi, "Approximation of solutions of the nonlinear Duffing equation involving both integral and non-integral forcing terms with separated boundary conditions," Computer Physics Communications, vol. 179, no. 6, pp. 409-416, 2008.

[4] B. Ahmad, "On the existence of p-periodic solutions for Duffing type integro-differential equations with p-Laplacian," Lobachevskii Journal of Mathematics, vol. 29, no. 1, pp. 1-4, 2008.

[5] Y. K. Chang and J. J. Nieto, "Existence of solutions for impulsive neutral integro-differential inclusions with nonlocal initial conditions via fractional operators," Numerical Functional Analysis and Optimization, vol. 30, no. 3-4, pp. 227-244, 2009.

[6] Z. Luo and J. J. Nieto, "New results for the periodic boundary value problem for impulsive integro-differential equations," Nonlinear Analysis: Theory, Methods \& Applications, vol. 70, no. 6, pp. 2248-2260, 2009.

[7] T. Jankowski, "Differential equations with integral boundary conditions," Journal of Computational and Applied Mathematics, vol. 147, no. 1, pp. 1-8, 2002.

[8] G. Wang, G. Song, and L. Zhang, "Integral boundary value problems for first order integro-differential equations with deviating arguments," Journal of Computational and Applied Mathematics, vol. 225, no. 2, pp. 602-611, 2009.

[9] K. Maleknejad, P. Torabi, and R. Mollapourasl, "Fixed point method for solving nonlinear quadratic Volterra integral equations," Computers \& Mathematics with Applications, vol. 62, no. 6, pp. 2555-2566, 2011.

[10] K. Maleknejad and M. N. Sahlan, "The method of moments for solution of second kind Fredholm integral equations based on B-spline wavelets," International Journal of Computer Mathematics, vol. 87, no. 7, pp. 1602-1616, 2010.

[11] K. Maleknejad, K. Nouri, and R. Mollapourasl, "Existence of solutions for some nonlinear integral equations," Communications in Nonlinear Science and Numerical Simulation, vol. 14, no. 6, pp. 2559-2564, 2009.

[12] W. Volk, "The iterated Galerkin method for linear integrodifferential equations," Journal of Computational and Applied Mathematics, vol. 21, no. 1, pp. 63-74, 1988.

[13] J. Banaś and K. Goebel, Measures of Noncompactness in Banach Spaces, vol. 60, Marcel Dekker, New York, NY, USA, 1980.

[14] A. Mohsen and M. El-Gamel, "A sinc-collocation method for the linear Fredholm integro-differential equations," Journal of Applied Mathematics and Physics, vol. 58, no. 3, pp. 380-390, 2007.

[15] A. Avudainayagam and C. Vani, "Wavelet-Galerkin method for integro-differential equations," Applied Numerical Mathematics, vol. 32, no. 3, pp. 247-254, 2000.

[16] F. Stenger, Numerical Methods Based on Sinc and Analytic Functions, vol. 20, Springer, New York, NY, USA, 1993.

[17] L. Zhang, "Boundary value problem for first order impulsive functional integro-differential equations," Journal of Computational and Applied Mathematics, vol. 235, no. 8, pp. 2442-2450, 2011.

[18] G. Wang, L. Zhang, and G. Song, "Extremal solutions for the first order impulsive functional differential equations with 
upper and lower solutions in reversed order," Journal of Computational and Applied Mathematics, vol. 235, no. 1, pp. 325-333, 2010.

[19] M. Dehghan and R. Salehi, "The numerical solution of the non-linear integro-differential equations based on the meshless method," Journal of Computational and Applied Mathematics, vol. 236, no. 9, pp. 2367-2377, 2012. 


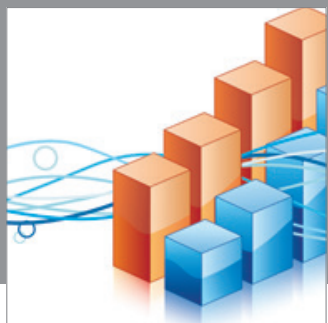

Advances in

Operations Research

mansans

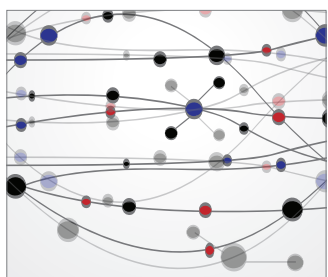

The Scientific World Journal
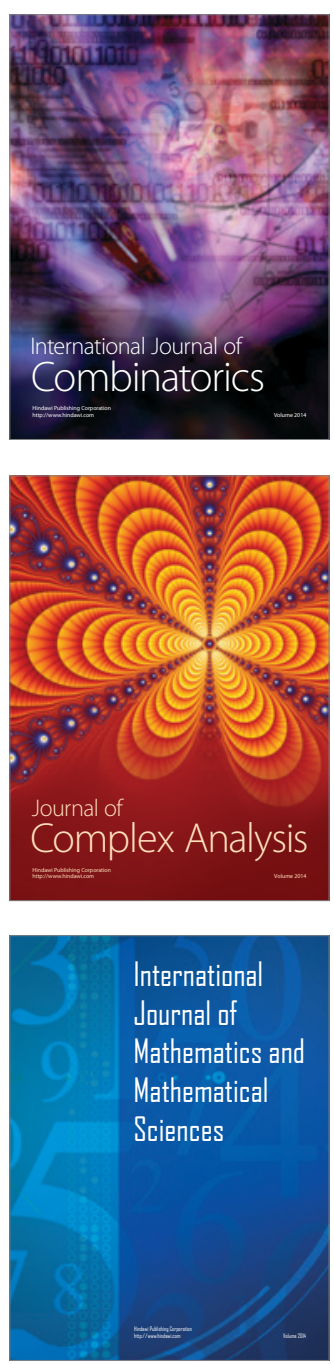
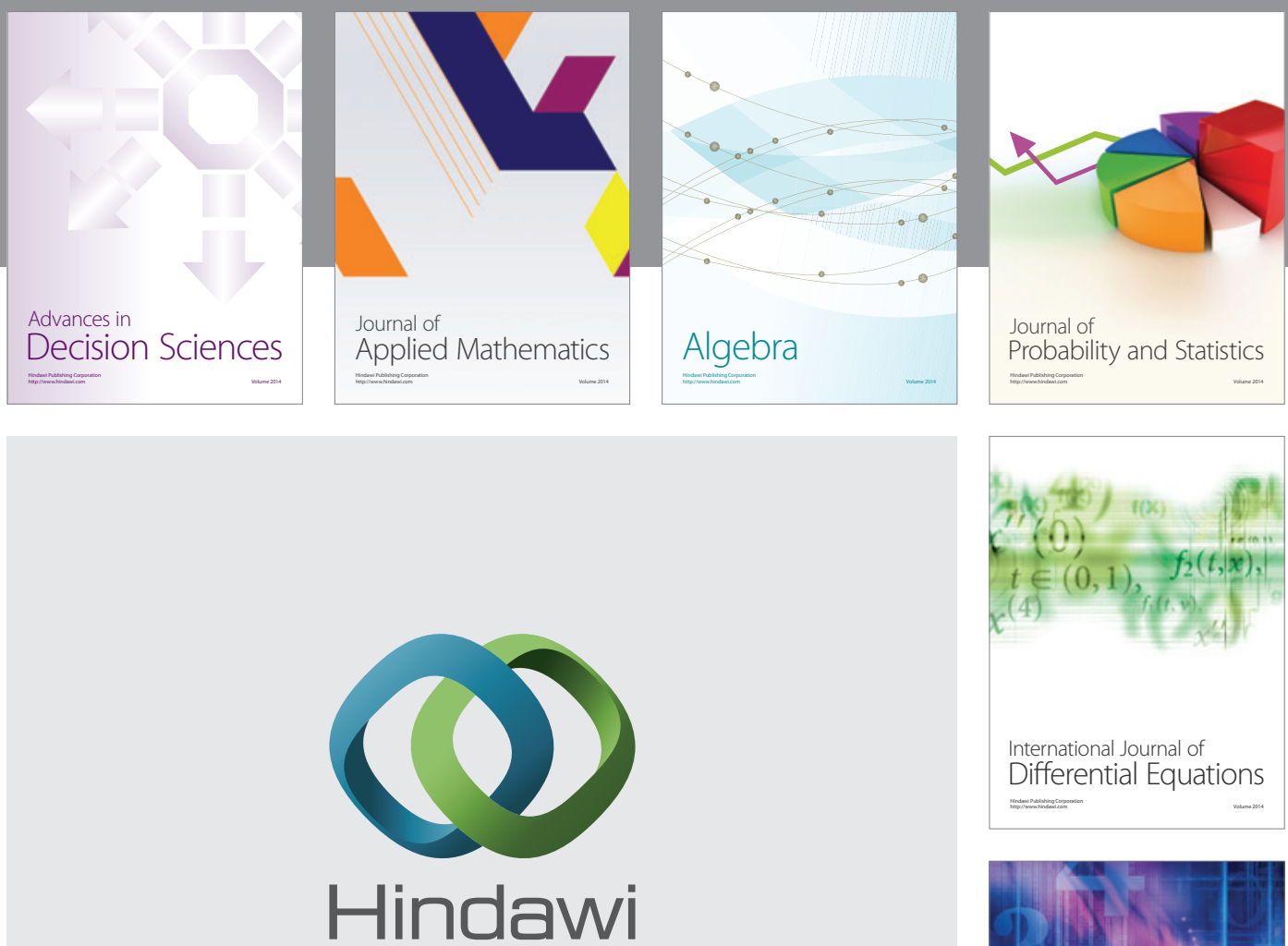

Submit your manuscripts at http://www.hindawi.com
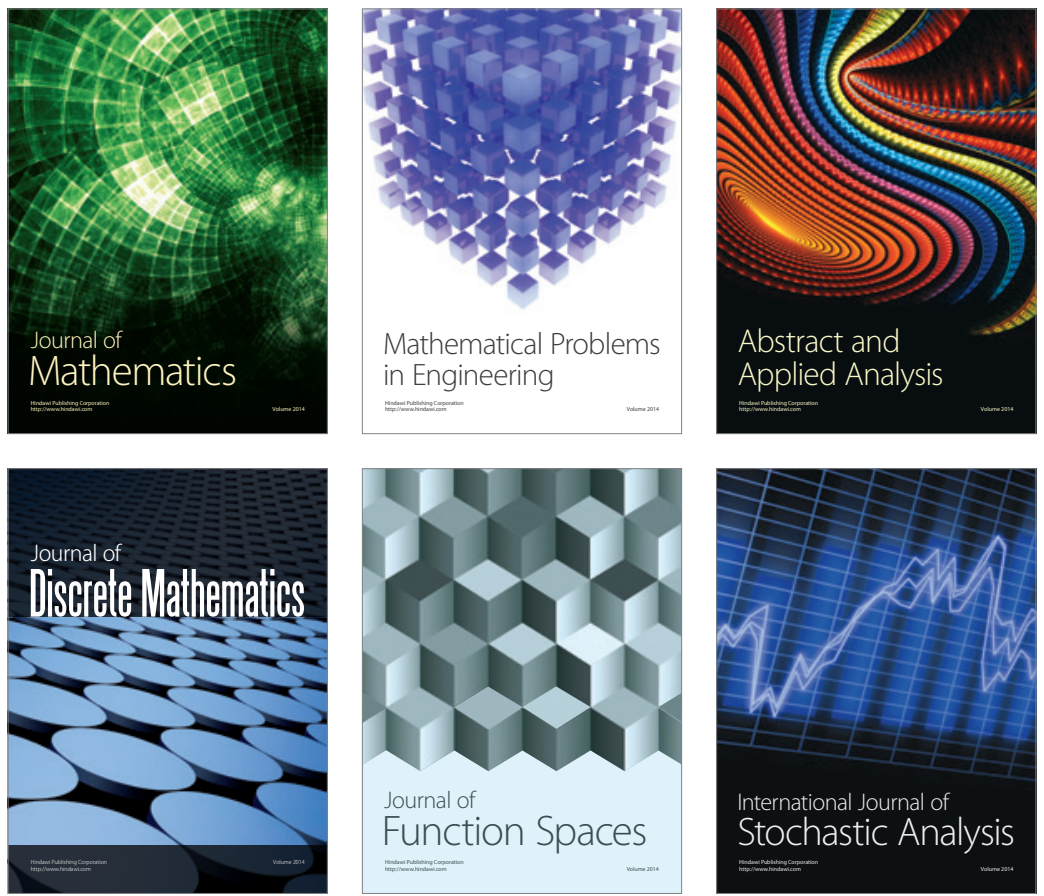

Journal of

Function Spaces

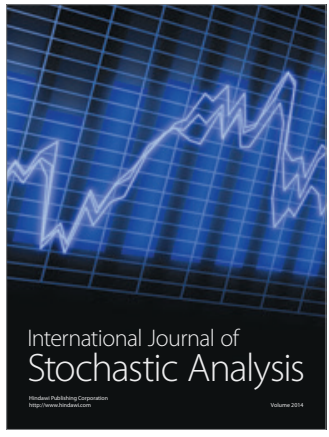

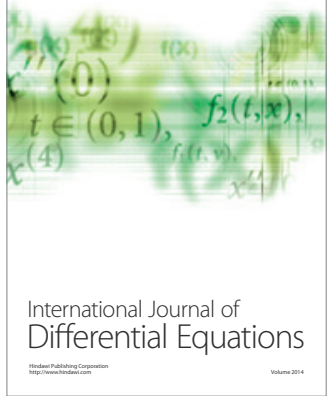
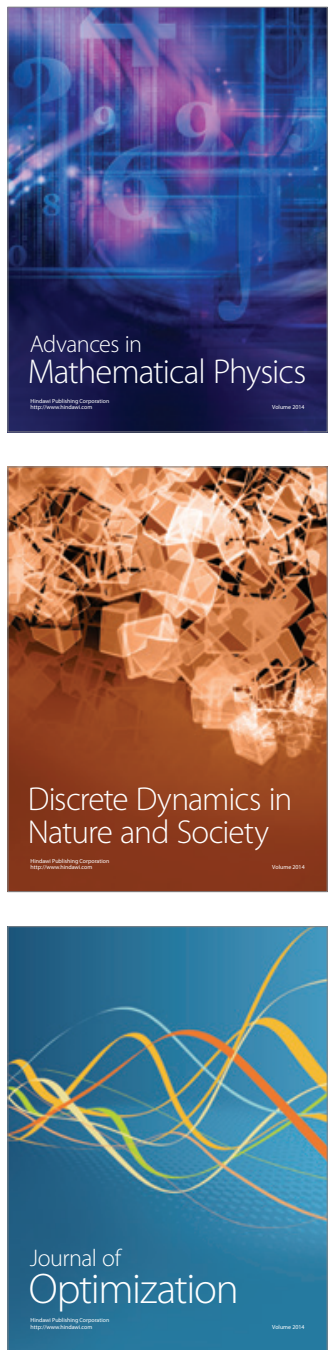